Review

\title{
Thyroid Dysfunction: Risk and Management in Dentistry
}

\author{
Georges Aoun \\ Department of Oral Medicine and Maxillofacial Radiology, Faculty of Dental Medicine, Lebanese University, Lebanon
}

\section{Article history}

Received: 28-01-2021

Revised: 10-03-2021

Accepted: 11-03-2021

Email: dr.georgesaoun@gmail.com

\begin{abstract}
The thyroid gland secretes essential hormones for the growth regulation and the metabolic activity of the body. Thyroid dysfunction includes many conditions that can indirectly affect dental treatments. Therefore, the practitioner must be aware of these pathologies and their signs and symptoms in order to elaborate specific dental treatment plans, thus avoiding any per-and/or postoperative complications. Moreover, the dentist can help in the screening of undiagnosed thyroid dysfunction among patients.
\end{abstract}

Keywords: Dentistry, Hyperthyroidism, Hypothyroidism, Management, Oral Manifestations, Thyroid Gland

\section{Introduction}

The thyroid is an endocrine gland located on the anterior midline of the neck below the inferior part of the larynx at the superior segment of the trachea. It consists of two oval shaped lobes connected by an isthmus. The mean lobe dimensions and weight are $5 \times 2.5 \times 2.5 \mathrm{~cm}$ and $25 \mathrm{mg}$, respectively (Khan et al., 2020). Microscopically, the thyroid gland is composed of spherical follicles bordered by follicular cells and containing a substance called colloid which is thyroglobulin. This thyroglobulin along with the circulating iodine form monoiodotyrosine and diiodotyrosine, precursors of the thyroid hormones, tetraiodothyronine known as Thyroxine (T4) and Triiodothyronine (T3) (Khan et al., 2020).

Between the thyroid follicles or inside their wall are located multiple small cells, the C-cells (parafollicular cells), responsible for another thyroid hormone known as calcitonin (Khan et al., 2020).

The secretion of thyroid hormones, mainly T3 and $\mathrm{T} 4$, is directly under the control of the hypothalamus via the TSH releasing hormone-TRH and the pituitary gland via the thyroid stimulating hormone-TSH. This is done through a negative feedback mechanism (Ortiga-Carvalho et al., 2011).

As for calcitonin secretion, it is mostly stimulated by the increase of serum calcium concentration (Felsenfeld and Levine, 2015).

The thyroid hormones are essential for the metabolic activity of all the body; they are essential to the normal functioning of most of the organs. Some of these functions are summarized in Table 1.

Table 1: Some of the thyroid functions (Khan et al., 2020)

They assist in the growth, development and differentiation of all the body cells (Hofstee et al., 2019; Talat et al., 2019).

They are a key regulator of the Basal Metabolic Rate (BMR) which is one of the major components of energy expenditure. In humans, BMR decrease can lead to obesity (Khan et al., 2020; Taousani et al., 2017).

They play an important role in calcium homeostasis (calcitonin, which is stimulated by hypercalcemia, fights against it) (Khan et al., 2020; Felsenfeld and Levine, 2015) and bone mineral homeostasis and density (deposition of calcium and phosphate in the bones) (Khan et al., 2020).

They are vital for the development of central nervous system in children and brain maturation and function for life (Khan et al., 2020;

Zhu et al., 2018; Bernal, 2000).

They stimulate somatic and psychic growth (Khan et al., 2020) and have major influence on the mood and behavior (mental and behavioral disorders may occur with thyroid hormones imbalance) (Acar and Ulgen, 2020).

They play an important role in the normal function of the heart (rate and contraction) and vascular physiology (Delitala et al., 2019;

Udovcic et al., 2017).

They contribute to the control carbohydrate, fat, protein and vitamins metabolism (Damiano et al., 2017).

They aid in regulating the body temperature (increased level of thyroid hormones rises body temperature and the opposite is true)

(Khan et al., 2020).

They play a vital role in maintaining electrolyte balance (Khan et al., 2020).

They influence the oxygen consumption by the cells and tissues (McAninch et al., 2015).

They stimulate gastrointestinal motility (Lee et al., 2012).

They improve the sensitivity of the $\beta$-adrenergic receptors to catecholamines (Khan et al., 2020).

They improve mitochondrial metabolism (Khan et al., 2020).

They serve a main role in regulating erythropoiesis (Park et al., 2017). 


\section{Thyroid Dysfunction}

Thyroid dysfunction is quite common in the general population, especially among women; moreover, it has been suggested that the number of undetected cases of thyroid dysfunction may be twice compared to the detected ones (Gessl et al., 2013). Typically, thyroid dysfunction is divided into hyperthyroidism and hypothyroidism.

\section{Hypothyroidism}

Hypothyroidism is a decrease in thyroid gland function and thyroid hormone secretion. It is classified into 3 categories: (a) Primary (autoimmune, iatrogenic, transient and iodine deficiency), (b) secondary (pituitary failure and tumor) and (c) tertiary (hypothalamic failure) (Jefferys et al., 2015).

In the United States, Hashimoto's thyroiditis (autoimmune disease) is the most frequent cause of hypothyroidism, but, globally, nutritional iodine deficiency is the most common cause (Patil et al., 2020). Some of hypothyroidism causes are summarized in Table 2 .

It should be noted that subacute and postpartum thyroiditis are transient types; in fact, patients with subacute thyroiditis (de Quervain thyroiditis, other) go through different phases over a period of weeks to months. Initially, they present as hyperthyroid, followed by a hypothyroid phase before eventually returning to being euthyroid (Tabassom and Edens, 2020). As for postpartum thyroiditis, the clinical course of the disease is variable and may include hyperthyroidism or hypothyroidism with spontaneous resolution in 32 and $43 \%$ of cases, respectively, or hyperthyroidism followed by hypothyroidism with spontaneous resolution (25\%) (Stagnaro-Green, 2002). Approximately $30 \%$ of women affected will stay hypothyroid one year postpartum (Keely, 2011).

Clinically, the signs and symptoms can be mild and nonspecific and differs from one patient to another (Patil et al., 2020). When severe hypothyroidism occurs in childhood, it is referred to as cretinism; the major cause of cretinism is chronic iodine deficiency and it is, usually, presented as following: (a) Short stature/poor growth, (b) decreased activity, (b) frontal bossing, (c) tongue protrusion, (d) hoarse voice, (e) hypertelorism, (f) muscles weakness (hypotonia), (g) dry skin and (h) alopecia (Pinto and Glick, 2002).

In adults, typical features of hypothyroidism include enlarged gland, weight gain, depression, anxiety, psychosis, memory loss, dysarthria and voice changes, bradykinesia, lethargy, dry skin and hair, pallor and jaundice, blank facial expressions, macroglossia, bradycardia, constipation, asthenia and muscle cramps, cold intolerance, sleep disorders, menstrual cycle irregularities, galactorrhea (Patil et al., 2020; Garber et al., 2012). Patient can also present sleep apnea, hyponatremia, hypercholesterolemia, congestive heart failure, pericardial effusion and prolonged QT interval (Garber et al., 2012).

It is important to note that myxedema coma, an exceptional lethal condition, is a result of severely advanced hypothyroidism with loss of the adaptive mechanism to maintain homeostasis (Elshimy and Correa, 2020).

Diagnosis of hypothyroidism is based on signs and symptoms, a low serum level of $\mathrm{T} 4$ and a high serum TSH level (Garber et al., 2012).

Hypothyroidism is primarily treated with levothyroxine monotherapy (Jonklaas et al., 2014). Therapy should be monitored based on TSH serum levels which must be tested every 4 to 8 weeks until target is achieved; when stable, then the monitoring interval can be extended to 6 to 12 months (Garber et al., 2012).

\section{Hyperthyroidism}

Hyperthyroidism is a syndrome related to thyroid hormone production excess. Graves's disease and toxic multinodular goiter are the more frequent causes of hyperthyroidism; however, other etiologies may include: Toxic adenomas, subacute thyroiditis and iodine-induced hyperthyroidism (Jod-Basedow phenomenon) (Jefferys et al., 2015; Mathew and Rawla, 2020) Table 3.

Table 2: Main causes of hypothyroidism (Jefferys et al., 2015) Causes of hypothyroidism

\section{- Primary:}

- Autoimmune disease:

- Atrophic thyroiditis

- Hashimoto's thyroiditis (Patil et al., 2020)

- Iatrogenic:

- $\quad$ Radioactive iodine therapy (Husseni, 2016)

- Thyroidectomy

- Drugs (anti-thyroid, amiodarone, thalidomide, lithium...) (Taylor et al., 2018)

- Transient:

- Subacute thyroiditis

- Postpartum thyroiditis (Patil et al., 2020)

Iodine deficiency (Patil et al., 2020)

Secondary hypothyroidism:

- Pituitary failure

Pituitary tumor

Tertiary hypothyroidism:

- Hypothalamic failure

Table 3: Main causes of hyperthyroidism (Jefferys et al., 2015)

Causes of hyperthyroidism

-Autoimmune

- Grave's disease

-Toxic nodular goiter

-Toxic adenoma

-Subacute thyroiditis

-Iodine-induced hyperthyroidism

-Drug-induced (amiodarone, lithium) 
Hyperthyroidism pathophysiology depends on the etiology of the condition; in Grave's disease, the original cause is autoimmune where thyroid-stimulating immunoglobulins are produced and went to bind to the $\mathrm{TSH}$ receptor, thus mimicking the TSH effects. On the other hand, the toxic multinodular goiter which presents with palpable thyroid nodules lead to the production of excess thyroid hormone from autonomous ectopic tissue (Mathew and Rawla, 2020).

Unlike the toxic multinodular goiter which can be formed by multiple nodules, toxic adenoma classically presents with a solitary papillary nodule that also causes hyperthyroidism (Mathew and Rawla, 2020).

Clinically, hyperthyroidism signs and symptoms reflect the state of increased metabolic activity including weight loss despite an augmented appetite, heart palpitation, anxiety, tremors, weakness, diarrhea, heat intolerance, atrial fibrillation, congestive heart failure and shortness of breath (Mathew and Rawla, 2020).

Physical examination may reveal an enlarged gland in the case of goiter or one or more palpable nodules (De Leo et al., 2016).

Diagnosis of hyperthyroidism is based on TSH, T4 and T3 serum levels tests. Due to the negative feedback that T3 and T4 apply on the pituitary gland, elevated T3 and/or elevated T4 will cause decreased TSH production (Menconi et al., 2014).

Treatment of hyperthyroidism depends on the underlying cause. Usually 2 types of treatment are considered, symptomatic and basic. In the former, the physician prescribes drugs depending on the symptoms; e.g., beta-adrenergic antagonist or calcium channel blocker to control the palpitations, anxiety and tremor (Ross et al., 2016). As for the basic treatment, radioactive iodine therapy, thionamide therapy and subtotal thyroidectomy can be used. It is important to note that all of them predispose the patient to possible long-term hypothyroidism. Thus, clinical assessment and T4 monitoring are crucial for patients who undergo any of these treatments (Mathew and Rawla, 2020).

It is important to note that untreated hyperthyroidism can lead to a major, potentially life-threatening complication referred to as thyroid storm (thyrotoxic crisis). In this condition reflecting the advanced state of hyperthyroidism and requiring immediate attention, the patient suffers from tachycardia, increased gastrointestinal motility, diaphoresis, anxiety and fever (Mathew and Rawla, 2020).

\section{Dental Management of Patients with Thyroid Dysfunction}

In dentistry, the risk in treating patients with controlled thyroid dysfunction is not elevated. Contrariwise, undiagnosed or uncontrolled thyroid conditions are risky (Malamed, 2015). For that, the dentist should be alert for any warning signs of such dysfunction and then refer the patient for medical consultation before any dental treatment.

Following are common oral manifestations found in thyroid disorders as well as the precautions dental practitioners should take to avoid potential complications during or after dental treatments.

\section{Hypothyroidism}

Frequent oral findings in hypothyroidism include macroglossia, glossitis, dysgeusia, compromised periodontal health, salivary gland enlargement and delayed dental eruption and wound healing (Table 4) (Pinto and Glick, 2002; Young, 1989).

Before treating patients with hypothyroidism, a specialized consultation is mandatory for more information on their medical conditions (e.g., cardiovascular status, lethargy, etc.) and the treatment will be provided accordingly. Well-controlled patients require no special precautions while in uncontrolled patients elective treatments should be deferred (Patton, 2015).

Moreover, dentists should be aware that patients under levothyroxine may experience an increased response to drugs affecting the central nervous system function such as narcotics, tranquillizers and barbiturates. Their use should be avoided (Pinto and Glick, 2002; Patton, 2015).

\section{Hyperthyroidism}

Oral manifestations in hyperthyroidism may include increased susceptibility to caries, severe periodontal disease, extra glandular thyroid tissue enlargement (mostly in the posterior dorsum of the tongue, known as ectopic or lingual thyroid), maxillary or mandibular osteoporosis, accelerated dental eruption/premature loss of temporary teeth and burning mouth syndrome (Table 4) (Pinto and Glick, 2002).

As patients with hyperthyroidism are susceptible to cardiovascular diseases such as atrial dysrhythmias, it is essential that dentists address these patients' cardiac history by consulting their physicians before any invasive procedures. These procedures should be deferred in case of symptomatic patients (tachycardia, irregular pulse, sweating, hypertension, tremor, etc.) and uncontrolled status (Pinto and Glick, 2002).

The use of vasoconstrictors (epinephrine) deserves special consideration when treating patients with hyperthyroidism. Epinephrine can exacerbate symptoms of tachycardia, dyspnea and fatigue (Patton, 2015). Moreover, dentists should use a stress reduction protocol; they can prescribe drugs to control anxiety but some oral sedatives may potentiate antithyroid drugs (Patton, 2015). 
Table 4: Oral manifestations of hypothyroidism and hyperthyroidism

\begin{tabular}{ll}
\hline Hypothyroidism & Hyperthyroidism \\
\hline -Macroglossia & -Increased susceptibility to caries \\
-Glossitis & -Severe periodontal disease \\
-Dysgeusia & -Extraglandular thyroid tissue enlargement (mainly lingual thyroid) \\
-Compromised periodontal health & -Maxillary or mandibular osteoporosis \\
-Salivary gland enlargement & -Accelerated dental eruption/premature loss of temporary teeth \\
-Delayed dental eruption & -Burning mouth syndrome \\
-Delayed wound healing & \\
\hline
\end{tabular}

Table 5: Thyroid dysfunction-considerations for dental treatments (Pinto and Glick, 2002)

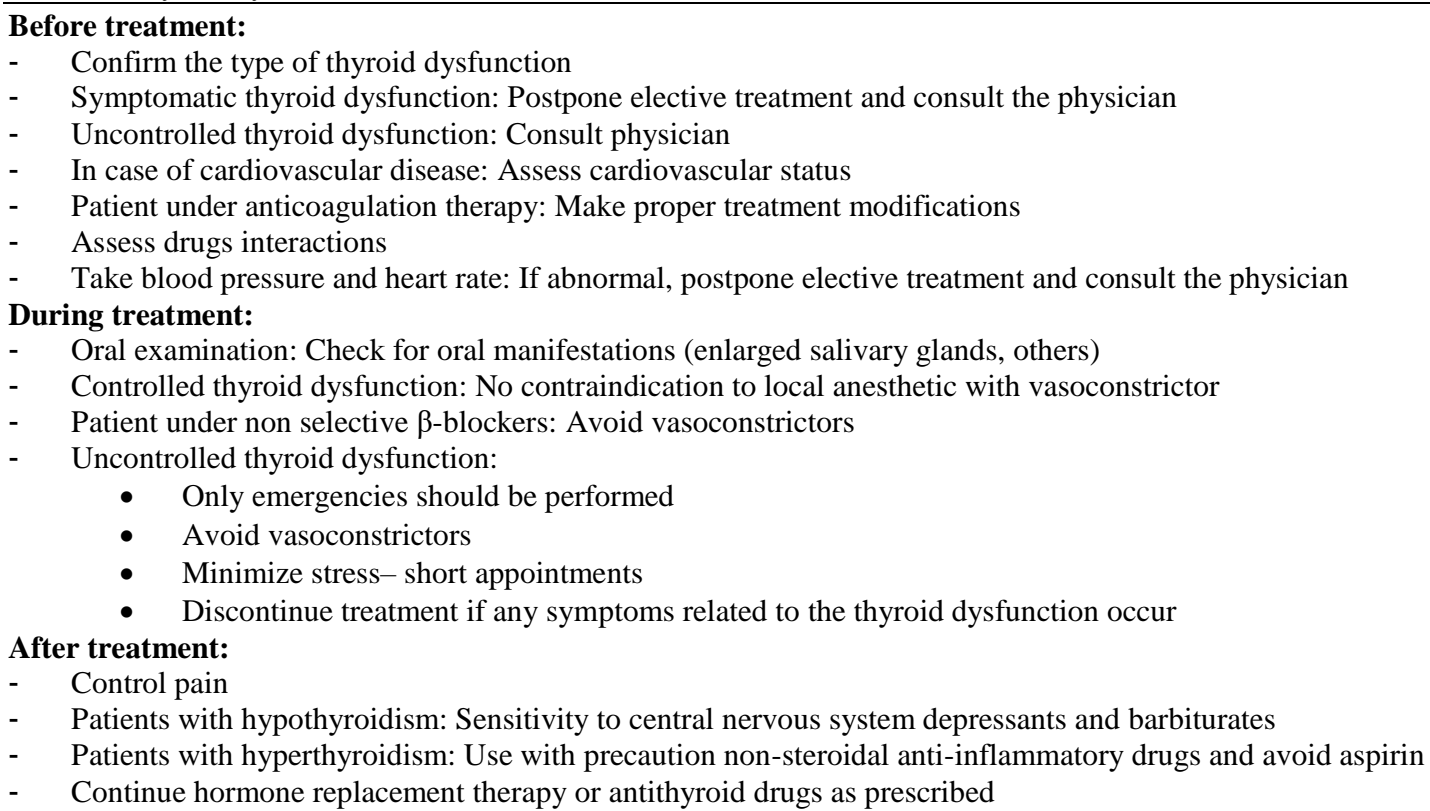

Finally, dentists should be prepared to take emergency measures while waiting the medical support if the thyrotoxic crisis clinical signs and symptoms occur. For that the following must be done: (a) Prevent hyperthermia by using cold towels to keep the patient cool, (b) administer intravenous hydrocortisone (100-300 $\mathrm{mg}$ ) and hypertonic glucose if available and (c) monitor vital signs and be prepared for cardiopulmonary resuscitation if necessary (Patton, 2015).

Table 5 resumes dental treatments modifications in case of thyroid dysfunction as proposed by (Pinto and Glick, 2002).

\section{Conclusion}

Thyroid disorders are serious conditions. Dental practitioners should be familiar with their oral and general manifestations so they can recognize any complication and evaluate their control level. In case of an undiagnosed/uncontrolled dysfunction, all elective dental treatment should be postponed until a full medical assessment is performed. Moreover, dentists should be prepared to take emergency measures and ask for medical support if the clinical manifestations of the thyrotoxic crisis occur.

\section{Ethics}

The author declares no potential conflicts of interest related to authorship and publication of this article.

\section{References}

Acar, H., \& Ülgen, A. (2020). Relationship between thyroid hormone levels and crime type: A controlled study in prisoners. International Journal of Endocrinology, 2020. https://www.hindawi.com/journals/ije/2020/9172134/

Bernal, J. (2000). Thyroid hormones in brain development and function. Endotext. South Dartmouth (MA). https://www.ncbi.nlm.nih.gov/books/NBK285549/

Damiano, F., Rochira, A., Gnoni, A., \& Siculella, L. (2017). Action of thyroid hormones, T3 and T2, on hepatic fatty acids: differences in metabolic effects and molecular mechanisms. International Journal of Molecular Sciences, 18(4), 744. https://www.mdpi.com/1422-0067/18/4/744 
De Leo, S., Lee, S. Y., \& Braverman, L. E. (2016). Hyperthyroidism. Lancet (London, England), 388(10047), 906-918 https://pubmed.ncbi.nlm.nih.gov/27038492/

Delitala, A. P., Scuteri, A., Maioli, M., Mangatia, P., Vilardi, L., \& Erre, G. L. (2019). Subclinical hypothyroidism and cardiovascular risk factors. Minerva Medica, 110(6), 530-545. https://europepmc.org/article/med/31726814

Elshimy, G., \& Correa, R. (2020) Myxedema. [Updated 2020 Oct 12]. In: StatPearls [Internet]. Treasure Island (FL): StatPearls Publishing. https://www.ncbi.nlm.nih.gov/books/NBK545193/

Felsenfeld, A. J., \& Levine, B. S. (2015). Calcitonin, the forgotten hormone: does it deserve to be forgotten?. Clinical Kidney Journal, 8(2), 180-187. https://academic.oup.com/ckj/article/8/2/180/471044 ?login=true

Garber, J. R., Cobin, R. H., Gharib, H., Hennessey, J. V., Klein, I., Mechanick, J. I., Pessah-Pollack, R., Singer, P. A., Woeber, K. A., \& American Association Of Clinical Endocrinologists And American Thyroid Association Taskforce On Hypothyroidism In Adults (2012). Clinical practice guidelines for hypothyroidism in adults: cosponsored by the American Association of Clinical Endocrinologists and the American Thyroid Association. Thyroid: official journal of the American Thyroid Association, 22(12), 1200-1235. Erratum in: Erratum in: Thyroid. 23(1): 129, \&Thyroid, 23(2); 251. https://pubmed.ncbi.nlm.nih.gov/22954017/

Gessl, A., Lemmens-Gruber, R., \& Kautzky-Willer, A. (2013). Thyroid disorders. Sex and Gender Differences in Pharmacology, 361-386. https://link.springer.com/chapter/10.1007/978-3642-30726-3_17

Hofstee, P., Bartho, L. A., McKeating, D. R., Radenkovic, F., McEnroe, G., Fisher, J. J., ... \& Cuffe, J. S. (2019). Maternal selenium deficiency during pregnancy in mice increases thyroid hormone concentrations, alters placental function and reduces fetal growth. The Journal of Physiology, 597(23), 5597-5617.

https://physoc.onlinelibrary.wiley.com/doi/abs/10.11 13/JP278473

Husseni, M. A. (2016). The incidence of hypothyroidism following the radioactive Iodine treatment of Graves' disease and the predictive factors influencing its development. World Journal of Nuclear Medicine, 15(1), 30-37. https://pubmed.ncbi.nlm.nih.gov/26912976/
Jefferys, A., Vanderpump, M., \& Yasmin, E. (2015). Thyroid dysfunction and reproductive health. The Obstetrician \& Gynaecologist, 17(1), 39-45. https://elearning.rcog.org.uk/sites/default/files/Recur rent\%20miscarriage/Jefferys_et_al-2015The_Obstetrician_\%26_Gynaecologist.pdf

Jonklaas, J., Bianco, A. C., Bauer, A. J., Burman, K. D., Cappola, A. R., Celi, F. S., ... \& Sawka, A. M. (2014). Guidelines for the treatment of hypothyroidism: prepared by the american thyroid association task force on thyroid hormone replacement. Thyroid, 24(12), 1670-1751.

Keely, E. J. (2011). Postpartum thyroiditis: an autoimmune thyroid disorder which predicts future thyroid health. Obstetric Medicine, 4(1), 7-11. https://journals.sagepub.com/doi/abs/10.1258/om.20 10.100041

Khan, Y.S., \& Farhana, A. (2020). Histology, Thyroid gland. [Updated 2020 Jul 3]. In: StatPearls [Internet]. Treasure Island (FL): StatPearls Publishing. https://www.ncbi.nlm.nih.gov/books/NBK551659/

Lee, J. H., Kwon, O. D., Ahn, S. H., Choi, K. H., Park, J. H., Lee, S., ... \& Jung, K. Y. (2012). Reduction of gastrointestinal motility by unilateral thyroparathyroidectomy plus subdiaphragmatic vagotomy in rats. World Journal of Gastroenterology: WJG, 18(33), 4570. https://www.ncbi.nlm.nih.gov/pmc/articles/PMC343 $5783 /$

Malamed, S. F. (2015). Medical emergencies in the dental surgery. Journal of the Irish Dental Association, 61(6).

Mathew, P., \& Rawla, P. (2020) Hyperthyroidism. [Updated 2020 Nov 21]. In: StatPearls [Internet]. Treasure Island (FL): StatPearls Publishing. https://www.ncbi.nlm.nih.gov/books/NBK537053/

McAninch, E. A., Miller, B. T., Ueta, C. B., Jo, S., \& Kim, B. W. (2015). Thyroid hormone at near physiologic concentrations acutely increases oxygen consumption and extracellular acidification in LH86 hepatoma cells. Endocrinology, 156(11), 4325-4335. https://academic.oup.com/endo/article/156/11/4325/ 2423195?login=true

Menconi, F., Marcocci, C., \& Marinò, M. (2014). Diagnosis and classification of Graves' disease. Autoimmunity Reviews, 13(4-5), 398-402. https://www.sciencedirect.com/science/article/abs/pi i/S1568997214000251

Ortiga-Carvalho, T. M., Chiamolera, M. I., Pazos-Moura, C. C., \& Wondisford, F. E. (2011). Hypothalamus-pituitary-thyroid axis. Comprehensive Physiology, 6(3), 1387-1428. 
Park, S., Han, C. R., Park, J. W., Zhao, L., Zhu, X., Willingham, M., ... \& Cheng, S. Y. (2017). Defective erythropoiesis caused by mutations Of the thyroid hormone receptor $\alpha$ gene. PLoS genetics, 13(9), e1006991. https://journals.plos.org/plosgenetics/article?rev=1\& id=10.1371/journal.pgen.1006991

Patil, N., Rehman, A., \& Jialal, I. (2020). Hypothyroidism. StatPearls [Internet]. https://www.ncbi.nlm.nih.gov/books/NBK519536/

Patton, L. L. (2015). The ADA practical guide to patients with medical conditions. John Wiley \& Sons.

Pinto, A., \& Glick, M. (2002). Management of patients with thyroid disease: oral health considerations. The Journal of the American Dental Association, 133(7), 849-858. https://www.sciencedirect.com/science/article/abs/pii/S 0002817714635297

Ross, D. S., Burch, H. B., Cooper, D. S., Greenlee, M. C., Laurberg, P., Maia, A. L., ... \& Walter, M. A. (2016). 2016 American Thyroid Association guidelines for diagnosis and management of hyperthyroidism and other causes of thyrotoxicosis. Thyroid, 26(10), 1343-1421. https://www.liebertpub.com/doi/abs/10.1089/thy. 2016.0229

Stagnaro-Green, A. (2002). Postpartum thyroiditis. The Journal of Clinical Endocrinology \& Metabolism, 87(9), 4042-4047. https://academic.oup.com/jcem/article/87/9/4042/ 2846380? $\log$ in=true

Tabassom, A., \& Edens, M. A. (2020) De Quervain Thyroiditis. [Updated 2020 Jul 15]. In: StatPearls [Internet]. Treasure Island (FL): StatPearls Publishing. https://www.ncbi.nlm.nih.gov/books/NBK526066/.
Talat, A., Khan, A. A., Nasreen, S., \& Wass, J. A. (2019). Thyroid Screening During Early Pregnancy and the Need for Trimester Specific Reference Ranges: A Cross-Sectional Study in Lahore, Pakistan. Cureus, 11(9). https://www.ncbi.nlm.nih.gov/pmc/articles/PMC6 823014/

Taousani, E., Savvaki, D., Tsirou, E., Poulakos, P., Mintziori, G., Zafrakas, M., ... \& Goulis, D. G. (2017). Regulation of basal metabolic rate in uncomplicated pregnancy and in gestational diabetes mellitus. Hormones, 16(3), 235-250. https://link.springer.com/article/10.1007/BF03401518

Taylor, P. N., Albrecht, D., Scholz, A., Gutierrez-Buey, G., Lazarus, J. H., Dayan, C. M., \& Okosieme, O. E. (2018). Global epidemiology of hyperthyroidism and hypothyroidism. Nature Reviews Endocrinology, 14(5), 301. https://www.nature.com/articles/nrendo.2018.18

Udovcic, M., Pena, R. H., Patham, B., Tabatabai, L., \& Kansara, A. (2017). Hypothyroidism and the heart. Methodist DeBakey Cardiovascular Journal, 13(2), 55 https://www.ncbi.nlm.nih.gov/pmc/articles/PMC5 512679/

Young, E. R. (1989). The thyroid gland and the dental practitioner. Journal (Canadian Dental Association), 55(11), 903-907. https://europepmc.org/article/med/2684364

Zhu, B., Zhao, G., Yang, L., \& Zhou, B. (2018). Tetrabromobisphenol A caused neurodevelopmental toxicity via disrupting thyroid hormones in zebrafish larvae. Chemosphere, 197, 353-361. https://www.sciencedirect.com/science/article/abs/pi i/S0045653518300882 